\title{
The Nonlinear Schroedinger Equation: Existence, Stability and Dynamics of Solitons
}

\author{
Vieri Benci * $\quad$ Marco Ghimenti ${ }^{\dagger} \quad$ Anna Maria Micheletti ${ }^{\ddagger}$
}

\begin{abstract}
In this paper we present some recent results concerning the existence, the stability and the dynamics of solitons occurring in the nonlinear Schroedinger equation when the parameter $h \rightarrow 0$.

We focus on the role played by the Energy and the Charge in the existence, the stability and the dynamics of solitons. Moreover, we show that, under suitable assumptions, the soliton approximately follows the dynamics of a point particle, namely, the motion of its barycenter $q(t)$ satisfies the equation
\end{abstract}

$$
\ddot{q}(t)+\nabla V(q(t))=H_{h}(t)
$$

where

$$
\sup _{t \in \mathbb{R}}\left|H_{h}(t)\right| \rightarrow 0 \quad \text { as } \quad h \rightarrow 0 .
$$

Mathematics subject classification. 35Q55, 35Q51, 37K40, 37K45, 47J35.

Keywords: Soliton dynamics, Nonlinear Schroedinger Equation, orbital stability, concentration phenomena, semiclassical limit.

\section{Introduction}

Roughly speaking a solitary wave is a solution of a field equation whose energy travels as a localized packet and which preserves this localization in time.

${ }^{*}$ Dipartimento di Matematica Applicata, Università degli Studi di Pisa, Via F. Buonarroti 1/c, Pisa, ITALY. e-mail: benci@dma.unipi.it

${ }^{\dagger}$ Dipartimento di Matematica e Applicazioni, Università degli Studi di Milano Bicocca, Via Cozzi, 53, Milano, ITALY. e-mail: marco.ghimenti@unimib.it

${ }^{\ddagger}$ Dipartimento di Matematica Applicata, Università degli Studi di Pisa, Via F. Buonarroti 1/c, Pisa, ITALY. e-mail: a.micheletti@dma.unipi.it 
By soliton we mean an orbitally stable solitary wave so that it has a particle-like behavior (for the definition of orbital stability we refer e.g. to Ref. [2, 3, 8, 14, 15, 24, 25]).

The aim of this paper is to review some recent results about the existence, the stability and the behavior of the solitary waves relative to the equation

$$
\left\{\begin{array}{l}
i h \frac{\partial \psi}{\partial t}=-\frac{h^{2}}{2} \Delta \psi+\frac{1}{2 h^{\alpha}} W^{\prime}(|\psi|) \frac{\psi}{|\psi|}+V(x) \psi \\
\psi(0, x)=\varphi(x)
\end{array}\right.
$$

where $\varphi(x)$ is a suitable initial data.

In the first section we examine the case $V \equiv 0$ and $h=1$ (see Ref. [3]). Under suitable assumption on $W$, there exists a stationary solution of the form $\psi(t, x)=U(x) e^{\frac{i}{h} \omega t}$, where $U$ is a radial function decaying at infinity which solves the equation

$$
-\Delta U+W^{\prime}(U)=2 \omega U
$$

This solution is found by a constrained minimization method that involves two prime integrals of the motion: the Charge and the Energy. By a concentration compactness argument it is proved that this stationary wave is stable, so this solution is a soliton.

In the second part, we consider $V \neq 0$ and $h$ small (see Ref. [4]). The stationary solution $U(x) e^{\frac{i}{h} \omega t}$ becomes

$$
\psi(t, x)=U\left(\frac{x}{h^{\beta}}\right) e^{\frac{i \omega t}{h^{\alpha+1}}}
$$

where

$$
\beta=1+\frac{\alpha}{2}
$$

If $\beta>0$, the stationary solution concentrate as $h \rightarrow 0$. Also, we can give a precise estimate of the behavior of the Energy and the Charge.

These estimates are the key ingredient to study the case $V \neq 0$. For $h$ sufficiently small, a solution of (10) with initial datum $\varphi(x)=\left[U\left(\frac{x-q_{0}}{h^{\beta}}\right)\right] e^{\frac{1}{h} \mathbf{v} \cdot x}$ is a soliton which travels like a point particle under the action of the potential $V$. In fact, in the last section of this review, we define the barycenter $q(t)$ of a soliton (see Ref. [4]) as

$$
q(t)=\frac{\int x|\psi(t, x)|^{2} d x}{\int|\psi(t, x)|^{2} d x}
$$


and we prove that it evolves approximatively like a point particle in a potential $V$. More exactly, $q(t)$ satisfies the Cauchy problem

$$
\left\{\begin{array}{l}
\ddot{q}(t)+\nabla V(q(t))=H_{h}(t) \\
q(0)=q_{0} \\
\dot{q}(0)=\mathbf{v}
\end{array}\right.
$$

where

$$
\sup _{t \in \mathbb{R}}\left|H_{h}(t)\right| \rightarrow 0 \text { as } h \rightarrow 0 .
$$

In the last years there are some result about existence and dynamics of soliton for the Nonlinear Schroedinger Equation (see, for example Ref. [6, 7, 10, 11, 17, 18, 21, 22,), in particular there are results which compare the motion of the soliton with the solution of the equation

$$
\ddot{X}(t)+\nabla V(X(t))=0
$$

for $t \in(0, T]$ for some constant $T<\infty$.

The result of Bronski and Jerrard [7] deals with a pure power nonlinearity and a bounded external potential. The authors have shown that if the initial data is close to $U\left(\frac{x-q_{0}}{h}\right) e^{i \frac{v_{0} \cdot c}{h}}$ in a suitable sense then the solution $\psi_{h}(t, x)$ of (11) satisfies for $t \in(0, T]$

$$
\left\|\frac{1}{h^{N}}\left|\psi_{h}(t, x)\right|^{2}-\left(\frac{1}{h^{N}} \int_{\mathbb{R}^{N}}\left|\psi_{h}(t, x)\right|^{2} d x\right) \delta_{X(t)}\right\|_{C^{1 *}} \rightarrow 0 \text { as } h \rightarrow 0 .
$$

Here $\delta_{X(t)}$ is the Dirac " $\delta$-function", $C^{1 *}$ is the dual of $C^{1}$ and $X(t)$ satisfies the equation (6) with $X(0)=q_{0}, \dot{X}(0)=v_{0}$.

In related papers of Keraani [17, 18] there are slight generalizations of the above result. Using a similar approach, Marco Squassina [22] and Alessandro Selvitella 21] described the soliton dynamics in an external magnetic potential.

Other results on this subject are in Ref. [10, 11]. In Ref. [10] the authors study the case of bounded external potential $V$.

In Ref. [11] the authors study the case of confining potential. They assume the existence of a stable ground state solution with a null space non degeneracy condition of the equation

$$
-\Delta \eta_{\mu}+\mu \eta_{\mu}+W^{\prime}\left(\eta_{\mu}\right)=0 \text {. }
$$

The authors define a parameter $\varepsilon$ which depends on $\mu$ and on other parameters of the problem. Under suitable assumptions they prove that there exists 
$T>0$ such that, if the initial data $\psi^{0}(x)$ is very close to $e^{i p_{0} \cdot\left(x-a_{0}\right)+i \gamma_{0}} \eta_{\mu_{0}}(x-$ $\left.a_{0}\right)$ the solution $\psi(t, x)$ of problem $\left(P_{1}\right)$ with initial data $\psi^{0}$ is given by

$$
\psi(t, x)=e^{i p(t) \cdot(x-a(t))+i \gamma(t)} \eta_{\mu(t)}(x-a(t))+w(t)
$$

with $\|w\|_{H^{1}} \leq \varepsilon, \dot{p}=-\nabla V(a)+o\left(\varepsilon^{2}\right), \dot{a}=2 p+o\left(\varepsilon^{2}\right)$ with $0<t<\frac{T}{\varepsilon}$ for $\varepsilon$ small.

In our paper 4 we do not require the uniqueness of the ground state solution which is, in general, not easy to verify, and we formulate our result such that it holds for any time $t$.

\section{Main assumptions}

In all this paper we make the following assumptions:

(i) the problem (1) has a unique solution

$$
\psi \in C^{0}\left(\mathbb{R}, H^{2}\left(\mathbb{R}^{N}\right)\right) \cap C^{1}\left(\mathbb{R}, L^{2}\left(\mathbb{R}^{N}\right)\right)
$$

(sufficient conditions can be found in Kato [16], Cazenave [9], GinibreVelo [13]).

(ii) $W: \mathbb{R}^{+} \rightarrow \mathbb{R}$ is a $C^{3}$ function which satisfies:

$$
\begin{gathered}
W(0)=W^{\prime}(0)=W^{\prime \prime}(0)=0 \\
\left|W^{\prime \prime}(s)\right| \leq c_{1}|s|^{q-2}+c_{2}|s|^{p-2} \text { for some } 2<q \leq p<2^{*}=\frac{2 N}{N-2} . \\
W(s) \geq-c|s|^{\nu}, c \geq 0,2<\nu<2+\frac{4}{N} \text { and } s \text { large } \\
\exists s_{0} \in \mathbb{R}^{+} \text {such that } W\left(s_{0}\right)<0
\end{gathered}
$$

(iii) $V: \mathbb{R}^{N} \rightarrow \mathbb{R}$ is a $C^{2}$ function which satisfies the following assumptions:

$$
\begin{gathered}
V(x) \geq 0 \\
|\nabla V(x)| \leq V(x)^{b} \text { for }|x|>R_{1}>1, b \in(0,1) \\
V(x) \geq|x|^{a} \text { for }|x|>R_{1}>1, a>1 .
\end{gathered}
$$

(iv) the main assumption

$$
\alpha>0
$$


Let us discuss the set of our assumptions:

The first assumption gives us the necessary regularity to define the barycenter and to prove that $q(t) \in C^{2}\left(\mathbb{R}, \mathbb{R}^{N}\right)$. The hypotheses on the nonlinearity are necessary in order to have a soliton type solutions. In particular, (12) is a standard requirement to have a smooth energy functional, (14) is the minimal requirement to have a focusing nonlinearity and (13) is necessary to have a good minimization problem to obtain the existence of a soliton. We require also that $V$ is a confining potential (assumption (iii)). This is useful on the last part of this paper, to prove the existence of a dynamics for the barycenter.

In our approach, the assumption $\alpha>0$ is crucial. In fact, as we will see in Section 3.1, the energy $E_{h}$ of a soliton $\psi$ is composed by two parts: the internal energy $J_{h}$ and the dynamical energy $G$. The internal energy is a kind of binding energy that prevents the soliton from splitting, while the dynamical energy is related to the motion and it is composed of potential and kinetic energy. We have that (see Section 4)

$$
J_{h}(\psi) \cong h^{N \beta-\alpha}
$$

and

$$
G(\psi) \cong\|\psi\|_{L^{2}}^{2} \cong h^{N \beta}
$$

Then, we have that

$$
\frac{G(\psi)}{J_{h}(\psi)} \cong h^{\alpha}
$$

So the assumption $\alpha>0$ implies that, for $h \ll 1, G(\psi) \ll J_{h}(\psi)$, namely the internal energy is bigger than the dynamical energy. This is the fact that guarantees the existence and the stability of the travelling soliton for any time. 


\section{$2.1 \quad$ Notations}

In the next we will use the following notations:

$$
\begin{aligned}
& \operatorname{Re}(z), \operatorname{Im}(z) \text { are the real and the imaginary part of } z \\
& B\left(x_{0}, \rho\right)=\left\{x \in \mathbb{R}^{N}:\left|x-x_{0}\right| \leq \rho\right\} \\
& S_{\sigma}=\left\{u \in H^{1}:\|u\|_{L^{2}}=\sigma\right\} \\
& J_{h}^{c}=\left\{u \in H^{1}: J_{h}(u)<c\right\} \\
& \partial_{t} \psi=\frac{\partial}{\partial t} \psi \\
& m=m_{\sigma^{2}}:=\inf _{u \in H^{1}, \int u^{2}=\sigma^{2}} J(u) \\
& \beta=1+\frac{\alpha}{2}
\end{aligned}
$$

\section{General features of NSE}

Equation (1) is the Euler-Lagrange equation relative to the Lagrangian density

$$
\mathcal{L}=\operatorname{Re}\left(i h \partial_{t} \psi \bar{\psi}\right)-\frac{h^{2}}{2}|\nabla \psi|^{2}-W_{h}(\psi)-V(x)|\psi|^{2}
$$

where, in order to simplify the notation we have set

$$
W_{h}(\psi)=\frac{1}{h^{\alpha}} W(|\psi|)
$$

Sometimes it is useful to write $\psi$ in polar form

$$
\psi(t, x)=u(t, x) e^{i S(t, x) / h}
$$

Thus the state of the system $\psi$ is uniquely defined by the couple of variables $(u, S)$. Using these variables, the action $\mathcal{S}=\int \mathcal{L} d x d t$ takes the form

$$
\mathcal{S}(u, S)=-\int\left[\frac{h^{2}}{2}|\nabla u|^{2}+W_{h}(u)+\left(\partial_{t} S+\frac{1}{2}|\nabla S|^{2}+V(x)\right) u^{2}\right] d x d t
$$

and equation (11) becomes:

$$
\begin{gathered}
-\frac{h^{2}}{2} \Delta u+W_{h}^{\prime}(u)+\left(\partial_{t} S+\frac{1}{2}|\nabla S|^{2}+V(x)\right) u=0 \\
\partial_{t}\left(u^{2}\right)+\nabla \cdot\left(u^{2} \nabla S\right)=0
\end{gathered}
$$




\subsection{The first integrals of NSE}

Noether's theorem states that any invariance under a one-parameter group of the Lagrangian implies the existence of an integral of motion (see e.g. Gelfand-Fomin[12]).

Now we describe the first integrals which will be relevant for this paper, namely the energy and the "hylenic charge".

Energy The energy, by definition, is the quantity which is preserved by the time invariance of the Lagrangian; it has the following form

$$
E_{h}(\psi)=\int\left[\frac{h^{2}}{2}|\nabla \psi|^{2}+W_{h}(\psi)+V(x)|\psi|^{2}\right] d x .
$$

Using (21) we get:

$$
E_{h}(u, S)=\int\left(\frac{h^{2}}{2}|\nabla u|^{2}+W_{h}(u)\right) d x+\int\left(\frac{1}{2}|\nabla S|^{2}+V(x)\right) u^{2} d x
$$

Thus the energy has two components: the internal energy (which, sometimes, is also called binding energy)

$$
J_{h}(u)=\int\left(\frac{h^{2}}{2}|\nabla u|^{2}+W_{h}(u)\right) d x
$$

and the dynamical energy

$$
G(u, S)=\int\left(\frac{1}{2}|\nabla S|^{2}+V(x)\right) u^{2} d x
$$

which is composed by the kinetic energy $\frac{1}{2} \int|\nabla S|^{2} u^{2} d x$ and the potential energy $\int V(x) u^{2} d x$.

Hylenic charge Following Ref. [2] the hylenic charge, is defined as the quantity which is preserved by by the invariance of the Lagrangian with respect to the action

$$
\psi \mapsto e^{i \theta} \psi
$$

For equation (11) the charge is nothing else but the $L^{2}$ norm, namely:

$$
\mathcal{H}(\psi)=\int|\psi|^{2} d x=\int u^{2} d x .
$$

Momentum If $V=0$ the Lagrangian is also invariant by translation. In this case we have the conservation of the momentum

$$
P_{j}(\psi)=h \operatorname{Im} \int \psi_{x_{j}} \bar{\psi} d x, \quad j=1,2,3
$$

hence we have the first Newton law for the barycenter. 


\section{The case $h=1, V=0$}

In this section we present some results contained in Ref. [3]. We minimize the internal energy $J(u)$ on the constraint $\left\{u \in H^{1}:\|u\|_{L^{2}}=\sigma\right\}$ for some $\sigma$ fixed. If $U$ is the minimizer and if $2 \omega$ is the Lagrange multiplier associated to $U, \psi(t, x)=U(x) e^{i \omega t}$ is a stationary solution of (10).

We get the following result

Lemma 1. Let $W$ satisfy (12), (13) and (14). Then, $\exists \bar{\sigma}$ such that $\forall \sigma>\bar{\sigma}$ there exists $\bar{u} \in H^{1}$ satisfying

$$
J(\bar{u})=m_{\sigma^{2}}:=\inf _{\left\{v \in H^{1},\|v\|_{L^{2}}=\sigma\right\}} J(v),
$$

with $\|\bar{u}\|_{L^{2}}=\sigma$. Then, there exist $\omega$ and $\bar{u}$ that solve (2), with $\omega<0$ and $\bar{u}$ positive radially symmetric.

In order to have stronger results, we can replace (14) with the following hypothesis

$$
W(s)<-s^{2+\epsilon}, 0<\epsilon<\frac{4}{N} \text { for small } s .
$$

In this case we find the following results concerning the existence of the minimizer of $J(u)$ for any $\sigma$.

Corollary 2. If (12), (13) and (30) hold, then for all $\sigma$, there exists $\bar{u} \in H^{1}$, with $\|\bar{u}\|_{L^{2}}=\sigma$, such that

$$
J(\bar{u})=\inf _{\left\{v \in H^{1},\|v\|_{L^{2}}=\sigma\right\}} J(v) .
$$

In particular, for $N=3$ we have

Corollary 3. Let $N=3$. If (12) and (13) hold and $W \in C^{3}$, with $W^{\prime \prime \prime}(0)<$ 0 , then for all $\sigma$, there exists $\bar{u} \in H^{1}$ with $\|\bar{u}\|_{L^{2}}=\sigma$ such that

$$
J(\bar{u})=\inf _{\left\{v \in H^{1},\|v\|_{L^{2}}=\sigma\right\}} J(v) .
$$

We sketch briefly the steps of the proof for Lemma 1.

Step 1: If $W$ satisfies (14) then $m_{\sigma^{2}}:=\inf _{S_{\sigma}} J(u)<0$

Step 2: If $W$ satisfies (13) then $m_{\sigma^{2}}>-\infty$, any minimizing Palais Smale sequence $u_{n}$ is bounded in $H^{1}$ and the Lagrange multipliers $\omega_{n}$ associated to $u_{n}$ are bounded in $\mathbb{R}$. 
Step 3: Any minimizing Palais Smale sequence converges in $H^{1}$ to a minimizer.

We point out that (14) is a fundamental requirement for the existence of a minimizer. In fact, if $W \geq 0$, then by Pohozaev identity we can prove that $U \equiv 0$ is the unique radial solution of (2).

Concerning the stability of stationary solution we set

$$
S=\left\{U(x) e^{i \theta}, \theta \in S^{1},\|U\|_{L^{2}}=\sigma, J(U)=m_{\sigma^{2}}\right\}
$$

Definition 4. $S$ is orbitally stable if

$$
\begin{gathered}
\forall \varepsilon, \exists \delta>0 \text { s.t. } \forall \varphi \in H^{1}\left(\mathbb{R}^{N}\right), \inf _{u \in S}\left\|\left|\psi_{0}\right|-u\right\|_{H^{1}}<\delta \Rightarrow \\
\forall t \inf _{u \in S}\||\psi(t, \cdot)|-u\|_{H^{1}}<\varepsilon
\end{gathered}
$$

where $\psi$ is the solution of (11) with initial data $\varphi$.

Using concentration compactness [19, 20] arguments we prove the following (see Ref. [3], Sect. 3)

Theorem 5. Let $W$ satisfy (12), (13) and (14). Then $S$ is orbitally stable.

This variational approach can be successfully used to find stable solitary waves for the nonlinear Klein Gordon equation

$$
\square \psi=W^{\prime}(|\psi|) \frac{\psi}{|\psi|} .
$$

Again, the crucial assumption to obtain solitons is (14) (see Ref. 1] for details).

We obtain a concentration result for a minimizer $U$ crucial for this work (see Ref. [4]).

Lemma 6. For any $\varepsilon>0$, there exists an $\hat{R}=\hat{R}(\varepsilon)$ and a $\delta=\delta(\varepsilon)$ such that, for any $u \in J^{m+\delta} \cap S_{\sigma}$, we can find a point $\hat{q}=\hat{q}(u) \in \mathbb{R}^{N}$ such that

$$
\frac{1}{\sigma^{2}} \int_{\mathbb{R}^{N} \backslash B(\hat{q}, \hat{R})} u^{2}(x) d x<\varepsilon .
$$

We give a sketch of the proof.

Proof. Firstly we prove that for any $\varepsilon>0$, there exists a $\delta$ such that, for any $u \in J^{m+\delta} \cap S_{\sigma}$, we can find a point $\hat{q}=\hat{q}(u) \in \mathbb{R}^{N}$ and a radial ground state solution $U$ such that

$$
\|u(x)-U(x-\hat{q})\|_{H^{1}} \leq \varepsilon .
$$


We argue by contradiction: if (34) do not hold, we can construct a minimizing sequence which not converge. At this point, given $\varepsilon$, there exist a point $\hat{q}=\hat{q}(u) \in \mathbb{R}^{N}$ and a radial ground state solution $U$ such that

$$
u(x)=U(x-\hat{q})+w \text { and }\|w\|_{H^{1}} \leq C \varepsilon .
$$

Now, we choose $\hat{R}$ such that

$$
\frac{1}{\sigma^{2}} \int_{\mathbb{R}^{N} \backslash B(0, \hat{R})} U^{2}(x) d x<C \varepsilon
$$

for all $U$ radial ground state solutions. This is possible because if $U$ is a minimizer for $J$ constrained on $S_{\sigma}$, then there exists two constants $C, R$, not depending on $U$ such that

$$
U(x)<C e^{-|x|} \text { for }|x|>>R .
$$

By this fact we get the claim.

We remark that, depending on the nonlinearity $W$, it is possible that the minimizer of the constrained problem is not unique. Anyway, by Lemma 6 , $\hat{R}$ does not depend on the minimizer.

\section{The case $h$ small enough}

We present now the main results contained in Ref. [4]. We recall some inequalities which are useful in the following. Let it be

$$
u(x):=v\left(\frac{x}{h^{\beta}}\right) .
$$

We have

$$
\|u\|_{L^{2}}^{2}=\int v\left(\frac{x}{h^{\beta}}\right)^{2} d x=h^{N \beta} \int v(\xi)^{2} d \xi=h^{N \beta}\|v\|_{L^{2}}^{2} .
$$

and

$$
\begin{aligned}
J_{h}(u) & =\int \frac{h^{2}}{2}|\nabla u|^{2}+\frac{1}{h^{\alpha}} W(u) d x= \\
& =\int \frac{h^{2}}{2}\left|\nabla_{x} v\left(\frac{x}{h^{\beta}}\right)\right|^{2}+\frac{1}{h^{\alpha}} W\left(v\left(\frac{x}{h^{\beta}}\right)\right) d x= \\
& =\int \frac{h^{N \beta+2-2 \beta}}{2}\left|\nabla_{\xi} v(\xi)\right|^{2}+h^{N \beta-\alpha} W(v(\xi)) d \xi= \\
& =h^{N \beta-\alpha} \int \frac{1}{2}\left|\nabla_{\xi} v(\xi)\right|^{2}+W(v(\xi)) d \xi=h^{N \beta-\alpha} J_{1}(v) .
\end{aligned}
$$


We give now some results about the concentration property of the solutions $\psi(t, x)$ of the problem (11). Given $K>0, h>0$, we put

$$
B_{h}^{K}=\left\{\begin{array}{c}
\varphi(x)=\psi(0, x)=u_{h}(0, x) e^{\frac{i}{h} S_{h}(0, x)} \\
\text { with } u_{h}(0, x)=\left[(U+w)\left(\frac{x}{h^{\beta}}\right)\right] \\
U \text { is a minimizer of } J \text { constrained on } S \sigma \\
\|U+w\|_{L^{2}}=\|U\|_{L^{2}}=\sigma \text { and } J(U+w) \leq m+K h^{\alpha} \\
\left\|\nabla S_{h}(0, x)\right\|_{L^{\infty}} \leq K \text { for all } h \\
\int_{\mathbb{R}^{N}} V(x) u_{h}^{2}(0, x) d x \leq K h^{N \beta-2 \alpha}
\end{array}\right\} .
$$

Considering the set $B_{h}^{K}$ as the admissible initial data set, we get

Theorem 7. Assume $V \in L_{\text {loc }}^{\infty}$ and (15). Fix $K>0, q \in \mathbb{R}^{N}$. Let $\alpha>0$.

For all $\varepsilon>0$, there exists $\hat{R}>0$ and $h_{0}>0$ such that, for any $\psi(t, x)$ solution of (1) with initial data $\psi(0, x) \in B_{h}^{K}$ with $h<h_{0}$, and for any $t$, there exists $\hat{q}_{h}(t) \in \mathbb{R}^{N}$ for which

$$
\frac{1}{\|\psi(t, x)\|_{L^{2}}^{2}} \int_{\mathbb{R}^{N} \backslash B\left(\hat{q}_{h}(t), \hat{R} h^{\beta}\right)}|\psi(t, x)|^{2} d x<\varepsilon .
$$

Here $\hat{q}_{h}(t)$ depends on $\psi(t, x)$.

We give the proof because it is simple and quite interesting.

Proof. By the conservation law, the energy $E_{h}(\psi(t, x))$ is constant with respect to $t$. Then we have

$$
\begin{aligned}
E_{h}(\psi(t, x)) & =E_{h}(\psi(0, x)) \\
& =J_{h}\left(u_{h}(0, x)\right)+\int_{\mathbb{R}^{N}} u_{h}^{2}(0, x)\left[\frac{\left|\nabla S_{h}(0, x)\right|^{2}}{2}+V(x)\right] d x \\
& \leq J_{h}\left(u_{h}(0, x)\right)+\frac{K}{2} \sigma^{2} h^{N \beta}+K h^{N \beta} \\
& =h^{N \beta-\alpha} J(U+w)+C h^{N \beta}
\end{aligned}
$$

where $C$ is a suitable constant. Now, by rescaling, and using that $\psi(0, x) \in$ $B_{h}^{K, q}$, we obtain

$$
\begin{aligned}
E_{h}(\psi(t, x)) & \leq h^{N \beta-\alpha} J(U+w)+C h^{N \beta} \\
& \leq h^{N \beta-\alpha}\left(m+K h^{\alpha}\right)+C h^{N \beta} \\
& =h^{N \beta-\alpha}\left(m+K h^{\alpha}+C h^{\alpha}\right)=h^{N \beta-\alpha}\left(m+h^{\alpha} C_{1}\right)
\end{aligned}
$$


where $C_{1}$ is a suitable constant. Thus

$$
\begin{aligned}
J_{h}\left(u_{h_{n}}(t, x)\right) & =E_{h}(\psi(t, x))-G(\psi(t, x) \\
& =E_{h}(\psi(t, x))-\int_{\mathbb{R}^{N}}\left[\frac{\left|\nabla S_{h}(t, x)\right|^{2}}{2}+V(x)\right] u_{h}(t, x)^{2} d x \\
& \leq h^{N \beta-\alpha}\left(m+h^{\alpha} C_{1}\right)
\end{aligned}
$$

because $V \geq 0$. By rescaling the inequality (41) we get

$$
J\left(u_{h}\left(t, h^{\beta} x\right)\right) \leq m+h^{\alpha} C_{1}
$$

So, if $\alpha>0$, for $h$ small by a simple argument and Lemma 6 we get the claim.

Roughly speaking we have that $J_{h}(\psi) \cong h^{N \beta-\alpha}$ and $G(\psi) \cong h^{N \beta}$ and this is the key of the proof.

To simplify in the following we take an initial data of the type

$$
\varphi(x)=U\left(\frac{x-q}{h^{\beta}}\right) e^{i \mathbf{v} \cdot x},
$$

where $q, \mathbf{v}$ are fixed. Obviously $\varphi(x) \in B_{h}^{K}$ for some $K$.

\subsection{Existence and dynamics of barycenter}

We recall the definition of barycenter of $\psi$

$$
q_{h}(t)=\frac{\int_{\mathbb{R}^{N}} x|\psi(t, x)|^{2} d x}{\int_{\mathbb{R}^{N}}|\psi(t, x)|^{2} d x} .
$$

The barycenter is not well defined for all the functions $\psi \in H^{1}\left(\mathbb{R}^{N}\right)$. Thus we need the following result:

Theorem 8. Let $\psi(t, x)$ be a global solution of (11) such that $\psi(t, x) \in$ $C\left(\mathbb{R}, H^{2}\left(\mathbb{R}^{N}\right)\right) \cap C^{1}\left(\mathbb{R}, L^{2}\left(\mathbb{R}^{N}\right)\right)$ with initial data $\psi(0, x)$ such that

$$
\int_{\mathbb{R}^{N}}|x||\psi(0, x)|^{2} d x<+\infty .
$$

Then the map $q_{h}(t): \mathbb{R} \rightarrow \mathbb{R}^{N}$, given by $(44)$ is $C^{2}\left(\mathbb{R}, \mathbb{R}^{N}\right)$ and it holds

$$
\begin{gathered}
\dot{q}_{h}(t)=\frac{\operatorname{Im}\left(h \int_{\mathbb{R}^{N}} \bar{\psi}(t, x) \nabla \psi(t, x) d x\right)}{\|\psi(t, x)\|_{L^{2}}^{2}} . \\
\ddot{q}_{h}(t)=\frac{\int_{\mathbb{R}^{N}} V(x) \nabla|\psi(t, x)|^{2} d x}{\|\psi(t, x)\|_{L^{2}}^{2}} .
\end{gathered}
$$


We have the following corollary

Corollary 9. Assume (16) and the assumptions of the previous theorem; then

$$
\ddot{q}_{h}(t)=-\frac{\int_{\mathbb{R}^{N}} \nabla V(x)|\psi(t, x)|^{2} d x}{\|\psi(t, x)\|_{L^{2}}^{2}} .
$$

\section{The final result}

\subsection{Barycenter and concentration point}

We have two quantities which describe the properties of the travelling soliton: the concentration point $\hat{q}$ and the barycenter $q$. If we want to describe the particle-like behavior of the soliton the concentration point $\hat{q}$ seems to be the natural indicator: it localize at any time $t$ the center of a ball which contains the larger part of the soliton. Unfortunately we do not have any control on the smoothness of $\hat{q}(t)$ (indeed $\hat{q}$ is nor uniquely defined). The barycenter, at the contrary, for a very large class of solutions has the required regularity, and the equation (47) is very similar to the equation of the motion we want to obtain. In this paragraph, we estimate the distance between the concentration point and the barycenter of a solution $\psi(t, x)$ for a potential satisfying hypothesis (15) and (17), say a confining potential.

The assumption (17) is necessary if we want to identify the position of the soliton with the barycenter. Let us see why. Consider a soliton $\psi(x)$ and a perturbation

$$
\psi_{d}(x)=\psi(x)+\varphi(x-d), d \in \mathbb{R}^{N}
$$

Even if $\varphi(x) \ll \psi(x)$, when $d$ is very large, the "position" of $\psi(x)$ and the barycenter of $\psi_{d}(x)$ are far from each other. In Lemma 12, we shall prove that this situation cannot occur provided that (17) hold. In a paper [5] in preparation, we give a more involved notion of barycenter of the soliton and we will be able to consider other situations.

Hereafter, fixed $K>0$, we assume that $\psi(t, x)$ is a global solution of the Schroedinger equation (1), $\psi(t, x) \in C\left(\mathbb{R}, H^{1}\right) \cap C^{1}\left(\mathbb{R}, H^{-1}\right)$, with initial data $\psi(0, x) \in B_{h}^{K}$ with $B_{h}^{K}$ given by (38). We start with some technical lemma.

Lemma 10. There exists a constant $L>0$ such that

$$
0 \leq \frac{1}{h^{N \beta-2 \alpha}} \int_{\mathbb{R}^{N}} V(x) u_{h}^{2}(t, x) d x \leq L \quad \forall t \in \mathbb{R} .
$$


The proof follows by estimating the energy.

Lemma 11. There exists a constant $K_{1}$ such that

$$
\left|q_{h}(t)\right| \leq K_{1} \text { for } t \in \mathbb{R} .
$$

The proof follows by Lemma 10 and by (17). Furthermore, we can choose $R_{2}$ such that

$$
\frac{\int_{|x| \geq R_{2}} u_{h}^{2}(t, x) d x}{\int_{\mathbb{R}^{N}} u_{h}^{2}(t, x) d x} \leq \frac{1}{2}
$$

Lemma 12. Given $0<\varepsilon<1 / 2$, and $R_{2}$ as in the previous lemma.

We get

1. $\sup _{t \in \mathbb{R}}\left|\hat{q}_{h}(t)\right|<R_{2}+\hat{R}(\varepsilon) h^{\beta}<R_{2}+1$, for all $h<\bar{h}$ and $\delta<\bar{\delta}$ small enough.

2. $\sup _{t \in \mathbb{R}}\left|q_{h}(t)-\hat{q}_{h}(t)\right|<\frac{3 L}{\sigma^{2} R_{3}^{a-1}}+3 R_{3} \varepsilon+\hat{R}(\varepsilon) h^{\beta}$, for any $R_{3} \geq R_{2}$, and for all $h$ small enough.

The hardest part of the proof is the estimate of

$$
I_{1}=\frac{\left|\int_{\mathbb{R}^{N} \backslash B\left(0, R_{3}\right)}\left(x-\hat{q}_{h}(t)\right) u_{h}^{2}(t, x) d x\right|}{\int_{\mathbb{R}^{N}} u_{h}^{2}(t, x) d x} .
$$

Using (17) and the previous estimates we can conclude.

We notice that $R_{1}, R_{2}$ and $R_{3}$ defined in this section do not depend on $\varepsilon$.

\subsection{Equation of the travelling soliton}

We prove that the barycenter dynamics is approximatively that of a point particle moving under the effect of an external potential $V(x)$.

Theorem 13. Assume (i)-(iv). Given $K>0$, let $\psi(t, x) \in C\left(\mathbb{R}, H^{2}\right) \cap$ $C^{1}\left(\mathbb{R}, H^{1}\right)$ be a global solution of equation (11), with initial data in $B_{h}^{K}, h<$ $h_{0}$. Then we have

$$
\ddot{q}_{h}(t)+\nabla V\left(q_{h}(t)\right)=H_{h}(t)
$$

with $\left\|H_{h}(t)\right\|_{L^{\infty}}$ goes to zero when $h$ goes to zero. 
Proof. We know by Theorem 8 , that

$$
\ddot{q}_{h}(t)+\frac{\int_{\mathbb{R}^{N}} \nabla V(x) u_{h}^{2}(t, x) d x}{\int_{\mathbb{R}^{N}} u_{h}^{2}(t, x) d x}=0
$$

Hence we have to estimate the function

$$
H_{h}(t)=\left[\nabla V\left(\hat{q}_{h}(t)\right)-\nabla V\left(q_{h}(t)\right)\right]+\frac{\int_{\mathbb{R}^{N}}\left[\nabla V(x)-\nabla V\left(\hat{q}_{h}(t)\right)\right] u_{h}^{2}(t, x) d x}{\int_{\mathbb{R}^{N}} u_{h}^{2}(t, x) d x} .
$$

By Lemma 11 and Lemma 12 we get

$$
\begin{aligned}
\left|\nabla V\left(\hat{q}_{h}(t)\right)-\nabla V\left(q_{h}(t)\right)\right| \leq \max _{\substack{i, j=1, \ldots, N \\
|\tau| \leq K_{1}+R_{2}+1}}\left|\frac{\partial^{2} V(\tau)}{\partial x_{i} \partial x_{j}}\right|\left|\hat{q}_{h}(t)-q_{h}(t)\right| \leq \\
\leq M\left[\frac{3 L}{\sigma^{2} R_{3}^{a-1}}+3 R_{3} \varepsilon+\hat{R}(\varepsilon) h^{\beta}\right],
\end{aligned}
$$

for any $R_{3} \geq R_{2}$ and some $M>0$.

To estimate

$$
\frac{\int_{\mathbb{R}^{N}}\left[\nabla V(x)-\nabla V\left(\hat{q}_{h}(t)\right)\right] u_{h}^{2}(t, x) d x}{\int_{\mathbb{R}^{N}} u_{h}^{2}(t, x) d x}
$$

we split the integral three parts.

$$
\begin{aligned}
L_{1} & =\frac{\int_{B\left(\hat{q}_{h}(t), \hat{R}(\varepsilon) h^{\beta}\right)}\left|\nabla V(x)-\nabla V\left(\hat{q}_{h}(t)\right)\right| u_{h}^{2}(t, x) d x}{\int_{\mathbb{R}^{N}} u_{h}^{2}(t, x) d x} ; \\
L_{2} & =\frac{\int_{\mathbb{R}^{N} \backslash B\left(\hat{q}_{h}(t), \hat{R}(\varepsilon) h^{\beta}\right)}|\nabla V(x)| u_{h}^{2}(t, x) d x}{\int_{\mathbb{R}^{N}} u_{h}^{2}(t, x) d x} ; \\
L_{3} & =\frac{\int_{\mathbb{R}^{N} \backslash B\left(\hat{q}_{h}(t), \hat{R}(\varepsilon) h^{\beta}\right)}\left|\nabla V\left(\hat{q}_{h}(t)\right)\right| u_{h}^{2}(t, x) d x}{\int_{\mathbb{R}^{N}} u_{h}^{2}(t, x) d x} .
\end{aligned}
$$

By the Theorem 7 and by Lemma 12 we have $L_{3}<M \varepsilon$.

We have also

$$
L_{1} \leq K_{1}+R_{2}+1 .
$$

Using hypothesis (16) we have

$$
L_{2} \leq M \varepsilon+\left[\frac{L}{\sigma^{2}}\right]^{b} \varepsilon^{1-b}
$$


where $b \in(0,1)$ is defined in (16) . Concluding we have

$$
\left|H_{h}(t)\right| \leq \frac{3 L M}{\sigma^{2} R_{3}^{a-1}}+\left[\frac{L}{\sigma^{2}}\right]^{b} \varepsilon^{1-b}+M\left(2+3 R_{3}\right) \varepsilon+2 M \hat{R}(\varepsilon) h^{\beta} .
$$

At this point we can have $\sup _{t}\left|H_{h}(t)\right|$ arbitrarily small choosing firstly $R_{3}$ sufficiently large, secondly $\varepsilon$ sufficiently small, and finally $h$ small enough.

Corollary 14. Let $\psi(t, x) \in C\left(\mathbb{R}, H^{2}\right) \cap C^{1}\left(\mathbb{R}, H^{1}\right)$ be a global solution of equation (11), with initial data $\varphi(x)=U\left(\frac{x-q_{0}}{h^{\beta}}\right) e^{\frac{i}{h} \mathbf{v} \cdot x}$ where $U$ is a radial minimizer of $J$ on $S \sigma, q_{0} \in \mathbb{R}^{N}, \mathbf{v} \in \mathbb{R}^{N}$, and $h<h_{0}$. Then the barycenter $q$ satisfies the following Cauchy problem

$$
\left\{\begin{array}{l}
\ddot{q}_{h}(t)+\nabla V\left(q_{h}(t)\right)=H_{h}(t) \\
q_{h}(0)=q_{0} \\
\dot{q}_{h}(0)=\mathbf{v}
\end{array}\right.
$$

Proof. The initial data belongs to $B_{h}^{K}$ for some $K$. We apply the previous results to obtain the equation for $\ddot{q}$. The initial data $q(0)$ and $\dot{q}(0)$ are derived with a direct calculation.

\section{The swarm interpretation}

In this section we present a different point of view on our problem. Although this approach is non rigorous, it provides some physical intuitions which are inspiring for a better understanding of the general framework. We will suppose that the soliton is composed by a swarm of particles which follow the laws of classical dynamics given by the Hamilton-Jacobi equation. This interpretation will permit us to give an heuristic proof of the main result.

First of all let us write NSE with the usual physical constants $m$ and $\hbar$ :

$$
i \hbar \frac{\partial \psi}{\partial t}=-\frac{\hbar^{2}}{2 m} \Delta \psi+\frac{1}{2} W_{\hbar}^{\prime}(\psi)+V(x) \psi .
$$

Here $m$ has the dimension of mass and $\hbar$, the Plank constant, has the dimension of action.

In this case equations (23) and (24) become:

$$
\begin{gathered}
-\frac{\hbar^{2}}{2 m} \Delta u+\frac{1}{2} W_{\hbar}^{\prime}(u)+\left(\partial_{t} S+\frac{1}{2 m}|\nabla S|^{2}+V(x)\right) u=0 \\
\partial_{t}\left(u^{2}\right)+\nabla \cdot\left(u^{2} \frac{\nabla S}{m}\right)=0 .
\end{gathered}
$$


The second equation allows us to interpret the matter field to be a fluid composed by particles whose density is given by

$$
\rho_{\mathcal{H}}=u^{2}
$$

and which move in the velocity field

$$
\mathbf{v}=\frac{\nabla S}{m}
$$

So equation (55) becomes the continuity equation:

$$
\partial_{t} \rho_{\mathcal{H}}+\nabla \cdot\left(\rho_{\mathcal{H}} \mathbf{v}\right)=0
$$

If

$$
-\frac{\hbar^{2}}{2 m} \Delta u+\frac{1}{2} W_{\hbar}^{\prime}(u) \ll u,
$$

equation (54) can be approximated by the eikonal equation

$$
\partial_{t} S+\frac{1}{2 m}|\nabla S|^{2}+V(x)=0 .
$$

This is the Hamilton-Jacobi equation of a particle of mass $m$ in a potential field $V$.

If we do not assume (57), equation (58) needs to be replaced by

$$
\partial_{t} S+\frac{1}{2 m}|\nabla S|^{2}+V+Q(u)=0
$$

with

$$
Q(u)=\frac{-\left(\hbar^{2} / m\right) \Delta u+W_{\hbar}^{\prime}(u)}{2 u} .
$$

The term $Q(u)$ can be regarded as a field describing a sort of interaction between particles.

Given a solution $S(t, x)$ of the Hamilton-Jacobi equation, the motion of the particles is determined by Eq.(56).

\subsection{An heuristic proof}

In this section we present an heuristic proof of the main result. This proof is not at all rigorous, but it helps to understand the underlying Physics.

If we interpret $\rho_{\mathcal{H}}=u^{2}$ as the density of particles then

$$
\mathcal{H}=\int \rho_{\mathcal{H}} d x
$$


is the total number of particles. By (59), each of these particle moves as a classical particle of mass $m$ and hence, we can apply to the laws of classical dynamics. In particular the center of mass defined in (5) takes the following form:

$$
q(t)=\frac{\int x m \rho_{\mathcal{H}} d x}{\int m \rho_{\mathcal{H}} d x}=\frac{\int x \rho_{\mathcal{H}} d x}{\int \rho_{\mathcal{H}} d x} .
$$

The motion of the barycenter is not affected by the interaction between particles (namely by the term (59)), but only by the external forces, namely by $\nabla V$. Thus the global external force acting on the swarm of particles is given by

$$
\vec{F}=-\int \nabla V(x) \rho_{\mathcal{H}} d x .
$$

Thus the motion of the center of mass $q$ follows the Newton law

$$
\vec{F}=M \ddot{q},
$$

where $M=\int m \rho_{\mathcal{H}} d x$ is the total mass of the swarm; thus by (60), (61) and (62), we get

$$
\ddot{q}(t)=-\frac{\int \nabla V \rho_{\mathcal{H}} d x}{m \int \rho_{\mathcal{H}} d x}=-\frac{\int \nabla V u^{2} d x}{m \int u^{2} d x} .
$$

If we assume that the $u(t, x)$ and hence $\rho_{\mathcal{H}}(t, x)$ is concentrated in the point $q(t)$, we have that

$$
\int \nabla V u^{2} d x \cong \nabla V(q(t)) \int u^{2} d x
$$

and so, we get

$$
m \ddot{q}(t) \cong-\nabla V(q(t)) .
$$

Notice that the equation $m \ddot{q}(t)=-\nabla V(q(t))$ is the Newtonian form of the Hamilton-Jacobi equation (58).

\section{References}

[1] J. Bellazzini, V. Benci, C. Bonanno, and A.M. Micheletti, Solitons for the nonlinear Klein-Gordon equation, Adv. Nonlinear Stud., to appear

[2] J. Bellazzini, V. Benci, C. Bonanno, E. Sinibaldi, Hylomorphic solitons, preprint arXiv:0810.5079

[3] J. Bellazzini, V. Benci, M. Ghimenti, and A.M. Micheletti, On the existence of the fundamental eigenvalue of an elliptic problem in $\mathbb{R}^{\mathbb{N}}$, Adv. Nonlinear Stud. 7 (2007), no. 3, 439-458. 
[4] V. Benci, M. Ghimenti, and A.M. Micheletti, The Nonlinear Schroedinger equation: solitons dynamics, preprint arXiv:0812.4152.

[5] V. Benci, M. Ghimenti, and A.M. Micheletti, The Nonlinear Schroedinger equation: solitons dynamics in a bounded potential, work in preparation..

[6] H. Berestycki and P.-L. Lions, Nonlinear scalar field equations. I. Existence of a ground state, Arch. Rational Mech. Anal. 82 (1983), no. 4, $313-345$.

[7] J.C. Bronski and R.L. Jerrard, Soliton dynamics in a potential, Math. Res. Lett. 7 (2000), no. 2-3, 329-342.

[8] T. Cazenave and P.-L. Lions, Orbital stability of standing waves for some nonlinear Schrödinger equations, Comm. Math. Phys. 85 (1982), no. 4, 549-561.

[9] T. Cazenave, Semilinear Schrödinger equations, Courant Lecture Notes in Mathematics, vol. 10, New York University Courant Institute of Mathematical Sciences, New York, 2003.

[10] J. Fröhlich, S. Gustafson, B.L.G. Jonsson, and I.M. Sigal, Solitary wave dynamics in an external potential, Comm. Math. Phys. 250 (2004), no. $3,613-642$.

[11] J. Fröhlich, S. Gustafson, B.L.G. Jonsson, and I.M. Sigal, Long time motion of NLS solitary waves in a confining potential, Ann. Henri Poincaré 7 (2006), no. 4, 621-660.

[12] I.M. Gelfand, S.V. Fomin, Calculus of Variations, Prentice-Hall, Englewood Cliffs, N.J. 1963.

[13] J. Ginibre and G. Velo, On a class of nonlinear Schrödinger equations. II. Scattering theory, general case, J. Funct. Anal. 32 (1979), no. 1, $33-71$.

[14] M. Grillakis, J. Shatah, and W. Strauss, Stability theory of solitary waves in the presence of symmetry. I, J. Funct. Anal. 74 (1987), no. 1, 160-197.

[15] M. Grillakis, J. Shatah, and W. Strauss, Stability theory of solitary waves in the presence of symmetry. II, J. Funct. Anal. 94 (1990), no. 2, 308348. 
[16] T. Kato, Nonlinear Schrödinger equations, Schrödinger operators (S nderborg, 1988), Lecture Notes in Phys., vol. 345, Springer, Berlin, 1989, pp. 218-263.

[17] S. Keraani, Semiclassical limit of a class of Schroedinger equations with potential, Comm. Partial Diff. Eq. 27, (2002), 693-704.

[18] S. Keraani, Semiclassical limit of a class of Schroedinger equations with potential II, Asymptotic Analysis, 47, (2006), 171-186.

[19] P.-L. Lions, The concentration-compactness principle in the calculus of variations. The locally compact case. I, Ann. Inst. H. Poincaré Anal. Non Linéaire 1 (1984), no. 2, 109-145.

[20] P.-L. Lions, The concentration-compactness principle in the calculus of variations. The locally compact case. II, Ann. Inst. H. Poincaré Anal. Non Linéaire 1 (1984), no. 4, 223-283.

[21] A. Selvitella, Asymptotic evolution for the semiclassical nonlinear Schroedinger equation in presence of electric and magnetic fields, J. Diff. Eq., 245 (2008), no. 9, 2566-2584

[22] M. Squassina, Soliton dynamics for nonlinear Schroedinger equation with magnetic field, , to appear in Manuscripta Math.

[23] M. Struwe, A global compactness result for elliptic boundary value problems involving limiting nonlinearities, Math. Z. 187 (1984), no. 4, 511517.

[24] M.I. Weinstein, Modulational stability of ground states of nonlinear Schrödinger equations, SIAM J. Math. Anal. 16 (1985), no. 3, 472-491.

[25] M.I. Weinstein, Lyapunov stability of ground states of nonlinear dispersive evolution equations, Comm. Pure Appl. Math. 39 (1986), no. 1, $51-67$. 